\title{
ACTION FIBRINOLYTIQUE DU STAPHYLOCOQUE
}

PAR

André Gratia

$\Lambda$ insi que Gonzenbach (') l'avait déjà remarqué, l'action coagulante du Staphylocoque sur le plasma oxalaté donne un caillot qui se fibrinolyse plus ou moins vite selon l'espèce animale à laquelle appartient le plasma expérimenté. Un caillot de lapin, par exemple, séfibrinolyse en 48 heures environ, tandis qu'un caillot de chèvre.n'est complètement dissout qu'après 3 ou 4 jours.

J'ai constaté que c'est à l'égard du plasma de chien que l'action fibrinolytique du Staphylocoque est de beaucoup la plus intense.

Expérience I. - A 1 cc. de plasma oxalaté de chien, on ajoute $0.2 \mathrm{cc}$. d'émulsion staphylococcique; le caillot. massif mais encore mou qu'on obtient après 1 à $11 / 2$ heure d'incubation à $37^{\circ}$, n'a pas même le temps de s'affermir qu'il commence déjà à se fibrinolyser ; une heure plus tard environ, il est complètement réduit en fines parcelles. Le cycle complet de coagulation et de fibrinolyse du plasma de chien est généralement révolu au bout de 2 à 3 heurès.

Il semble exister un certain parallélisme entre l'action coagulante et l'action fibrinolytique du Staphylocoque. C'est le plasma de chien que ce microbe coagule le plus vite, puis ce sont successivement les plasmas de lapin, d'homme, de chèvre et de mouton. C'est dans le même ordre qu'il les fibrinolyse.

Le Staphylocoque ne fibrinolyse pas seulement le caillot qu'il a produit lui-même, mais encore le caillot résultant d'une coagulation normale, ainsi que le prouve l'expérience suivante :

EXPÉRIENCE. II. - On recalcifie à l'aide de 4 cc. d'EPCa ( $\left.{ }^{2}\right), 1$ cc. de plasma oxalaté à $1 \%$ de chien et on divise le mélange en deux portions. Vers la 30 me minute, au moment où les premiers filaments de fibrine apparaissent, on ajoute à l'un des tubes, $0.2 \mathrm{cc}$. d'émulsion staphylococcique ;

(1) Centralblalt f. Bakter, vol. LXXVIII, fasc. 2, p. 79.

(2) $\mathrm{EP} C \mathrm{Ca}=$ Eau physiologique contenant $0,35 \%$ de $\mathrm{Ca} \mathrm{Cl}$. 
le tube se coagule rapidement, on peut le retourner à la $33^{\text {me }}$ minute, l'autre seulement à la $39^{\mathrm{me}}$. Puis on place les deux caillots à $37^{\circ}$. Une heure et demie après, le caillot qui contenait du Staphylocoque emprisonné dans ses mailles est tout à fait fibrinolysé ; le caillot témoin est encore intact après deux jours.

Il est, d'autre part, facile de vérifier que du Staphylocoque qui vient de fibrinolyser un caillot est non seulement capable d'en fibrinolyser d'autres, mais a encore conservé inaltérées ses vertus coagulantes à l'égard du plasma oxalaté normal. Le même Staphylocoque est coagulant en présence de fibrinogène, fibrinolytique en présence de fibrine. Il y a là un phénomène fort comparable à l'action coagulante puis protéolytique que le ferment labique exerce sur le lait.

Quels sont les caractères du plasma oxalaté coagulé puis fibrinolysé par le Staphylocoque ? Lorsqu'on centrifuge le fibrinolysat, on obtient un liquide opalescent qui ne se coagule plus après recalcification, même si on l'additionne de cytozyme ou de thrombine. Il ne se coagule pas davantage par le chauffage à $56^{\circ}$. Il semble bien ne plus contenir de fibrinogène. Il est de plus très fortement anticoagulant : il empêche radicalement la coagulation du plasma oxalaté normal recalcifié et la coagulation du plasma oxalaté ou du fribrinogène par la thrombine. Que ce pouvoir anticoagulant provient bien de la digestion de la fibrine et non du développement du Staphylocoque, cela se déduit des deux expériences suivantes :

EXPÉRIENCE III. - On ensemence à l'aide de Staphylocoque un même volume respectivement de plasma oxalaté et de sérum de chien. Le microbe se développe également bien dans les deux milieux. Le plasma se coagule, puis se fibrinolyse et devient anticoagulant, tandis que le sérum, même après une incubation prolongée, ne le devient pas.

EXPÉRIENCE IV. - On place à $37^{\circ}$, deux tubes contenant le même plasma oxalaté de chien ensemencé de Staphylocoques. Saisissañt te moment où la fíbrinolyse des caillots formés va commencer, l'on défibrine l'un des tubes et le replace aussitôt à l'étuve à côté de l'autre. Dans celuici le caillot laissé à lui-mêmie ne tarde pas à se désagréger et à donner un fibrinolysat anticoagulant. A ce moment, nous voyons que dans le premier tube, il s'est reformé un nouveau caillot que nous soustrayons à la fibrinolyse par une nouvelle défibrination. Une heure après, nouveau caillot, beaucoup plus mou naturellement, que nous défibrinons encore et ainsi de suite à plusieurs reprises. Le plasma enfin complètement défibriné n'est pas anticoagulant. 
Cette expérience montre encore que, contrairement à ce qui se passe dans le plasma des autres animaux étudiés, dans le plasma de chien la fibrinolyse débute avant que tout le fibrinogène n'ait été coagulé ; elle anticipe sur la fin de la coagulation, les deux processus chevauchant. On pourrait donc imaginer des cas où cette interférence des deux processus serait beaucoup plus précoce, si précoce même que la fibrinolyse s'exerçant au fur et à mesure de la coagulation, celle-ci pourrait ne plus être apparente; il y aurait un moment où le plasma serait déjà devenu anticoagulant alors qu'il contient encore du fibrinogène. Cette formule correspond singulièrement à celle que nous a donnée le plasma de lapin rendu incoagulable et anticoagulant par l'action prolongée du Streptocoque.

Ce serait peut-être là, - pure suggestion d'ailleurs, - une façon d'expliquer les résultats paradoxaux que nous avons observés au cours de nos recherches sur ce dernier microbe.

Pourquoi la fibrinolyse straphylococcique s'exerce-t-elle si énergiquement sur le plasma de chien et si péniblement sur le plasma des autres animaux ? Celà tient-il à la nature du fibrinogène - le fibrinogène de chien serait-il plus fibrinolysable ? - ou, au contraire, aux autres éléments du plasma - le plasma de lapin, par exemple, possédant des propriétés inhibitrices que ne posséderait pas le plasma de chien ? C'est ce que des expériences en cours nous permettront de savoir.

Il importerait aussi d'établir si l'action fibrinolytique du Staphylocoque est liée à la vitalité de cet organisme, ou bien si des extraits stériles de ce microbe n'auraient pas la même propriété. 